\title{
Space charge limited flow of a thin electron beam confined by a strong magnetic field
}

\author{
A.Rokhlenko and J. L. Lebowitz* \\ Department of Mathematics, Rutgers University \\ Piscataway, NJ 08854-8019
}

\begin{abstract}
An approximate analytic theory is developed and implemented numerically for calculating the space charge limited current and electric field of a thin cylindrical beam or current sheet between two wide parallel electrodes. The flow is confined by a sufficiently strong magnetic field. Assuming that the potential and current density are almost homogeneous in the direction transversal to the flow we compute the beam current and profile by a variational method. We find that the average current density scales as the reciprocal of the beam width when the latter becomes very small. The total cylindrical beam current thus decreases proportionly to its diameter while the total current of a sheet becomes almost independent of the width in this regime.
\end{abstract}

PACS: 52.27.Jt; 52.59.Sa; 52.59.Wd; 85.45.Bz

\section{Introduction}

Space charge limited electron flow in two and three dimensions presents a difficult nonlinear mathematical problem whose solution is important for many practical applications. In the design of high power electron beams [1-4] the one dimensional Child-Langmuir limit (CLL) [5] has been a benchmark for almost a century but corrections have to be made for the "current wings" near the boundaries of the flow. Consequently the current of narrow beams [6-8] show great divergences from CLL.

In a previous article [9] we considered a planar emitting region whose width $2 a$ is much larger than the cathodeanode distance ( 1 in our dimensionless units). In the case of narrow beams, $2 a \ll 1$, considered here, the moderate variations of the potential in the transversal direction will be used to reduce the dimensionality of the problem. We assume that the two parallel electrodes are large enough for the electric field to be homogeneous far away from the beam. A magnetic field directed along the $y$-axis keeps the current, which is emitted either by a long narrow straight strip or a circular disk, perpendicular to the electrodes without spreading out as shown in Fig. 1.

The outline of the paper is as follows. In section 2 we set up the problem and solve the Laplace equation for the vacuum field outside the current sheet. This solution serves as a boundary condition to derive a closed equation for the potential and current in the space charge region using the continuity of the electric field. In section 3 we specialize to the case of narrow beams and make certain approximations. We then solve the approximate problem iteratively in section 4 using a direct variational method. The results are presented in section 5 . We also consider there the asymptotics of the current density in the limit $a \rightarrow 0$. In section 6 we extend our approach to cylindrical current beams. Section 7 is devoted to a discussion of results.

\footnotetext{
*Also Department of Physics
}

\section{Formulation of problem.}

We will use dimensionless units: linear sizes are measured as fractions of the inter-electrode distance $D$, the potential $\phi(x, y)$ is given as a fraction of the interelectrode voltage $V$, and the current density $j(x)$ is in the units of the Child-Langmuir current density $j_{0}=$ $V^{3 / 2} \sqrt{2 e} / 9 \pi D^{2} \sqrt{m}$, obtained in the limit $a=\infty[5] ; e$ and $m$ are the electron charge and mass.

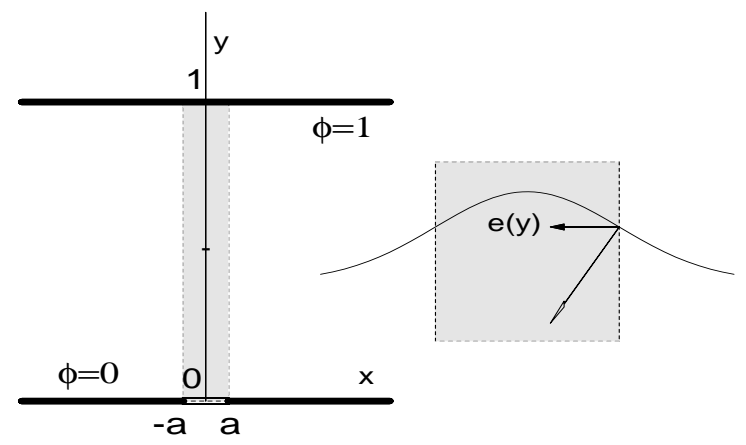

FIG. 1. Cross-section of the current with an expanded schematic graph of a contour of constant potential and electric field

The nonlinear Poisson equation which determines both the potential $\phi(x, y)$ between the electrodes and the current density $j(x)$ for a current sheet, see Fig. 1, has the form [9]

$$
\frac{\partial^{2} \phi}{\partial x^{2}}+\frac{\partial^{2} \phi}{\partial y^{2}}=\frac{4 j(x)}{9 \sqrt{\phi(x, y)}}
$$

Eq. (1) is to be solved with the boundary conditions

$$
\phi(x, 0)=0, \phi(x, 1)=1 \text {. }
$$

The unknown space charge limited current $j(x)$, in the region $|x|<a$, is to be determined from the condition

$$
\frac{\partial \phi}{\partial y}(x, 0)=0, \text { for }|x|<a .
$$


For $|x|>a, j(x)=0$ and (1) reduces to the Laplace equation in vacuum. We assume that (1) and (2) are well posed but leave a mathematical investigation of this point for the future.

A natural approach to solving (1) is to separate it into a standard boundary value problem for the Laplace equation outside the space charge region, $|x|>a$, and a nonlinear inner problem in the rectangle, $-a \leq x \leq$ $a, 0 \leq y \leq 1$. Taking the potential $\psi(y)=\bar{\phi}(a, y)$ on the sheet surface as given and using the symmetry $\phi(x, y)=\phi(-x, y)$ we find $\phi(x, y)$ for $x>a$. From this one calculates the external electric field in the $x$-direction $e_{+}(y)=-\frac{\partial \phi}{\partial x}(a, y)$ at $x=a_{+}$. The continuity of the potential and electric field at the space charge boundary gives the additional boundary conditions $\psi(y)=\phi(a, y)$ and $e_{-}(y)=e_{+}(y)=e(y)$ needed for solving (1) in the rectangle $|x|<a, 0 \leq y \leq 1$.

\section{Vacuum Fields}

We want to solve the Laplace equation (1) in the region $x>a$ with the boundary conditions,

$$
\phi(x, 0)=0, \phi(x, 1)=1, \phi(a, y)=\psi(y),
$$

and

$$
\phi(x, y) \rightarrow y \quad \text { for } \quad x \rightarrow \infty,
$$

expecting $\psi(y)$ to be monotone with finite first derivatives. Taking the potential in the form of a Fourier series

$$
\phi(x, y)=y+\frac{2}{\pi} \sum_{k=1}^{\infty} \frac{a_{k}}{k} e^{\pi k(a-x)} \sin \pi k y, x>a
$$

(3) is satisfied if

$$
a_{k}=\int_{0}^{1} \frac{d \psi}{d y}(y) \cos \pi k y d y .
$$

An illustrative (and useful for our computations) example is the case $\psi(y)=y^{4 / 3}$. Eq. (5) then gives

$$
a_{k}=\frac{4}{3} \Re\left[(-i k \pi)^{-4 / 3} \gamma\left(\frac{4}{3},-i k \pi\right)\right],
$$

where $\gamma(u, v)$ is the incomplete gamma function [10].

Using (4) and (5) the $x$-component of the electric field at the beam boundary $x=a$ can be written as

$$
e(y)=-\frac{\partial \phi}{\partial x}(a, y)=2 \sum_{k=1}^{\infty} a_{k} \sin \pi k y .
$$

Substituting (5) into (6) and summing up the series explicitly we get an alternative form for $e(y)$

$$
e(y)=\sin \pi y \int_{0}^{1} \frac{\psi^{\prime}(t)-\psi^{\prime}(y)}{\cos \pi t-\cos \pi y} d t .
$$

We note that Eq.(7) can also be derived by using conformal mapping, as in [9]. For $\psi(y)=y^{2}$ Eq.(7) becomes

$$
e(y)=\frac{4}{\pi} \int_{0}^{y} \ln \tan \frac{\pi t}{2} d t .
$$

\section{The inner region}

Eq. (1) together with boundary conditions (2) and (7) now define the space charge limited current problem in the rectangle $|x|<a, 0 \leq y \leq 1$, the shaded region in Fig. 1. Also shown there is an expanded cross section together with a schematic drawing of an equipotential surface and electric field vector with its $x$-component. As already noted the CLL potential $(a=\infty)$ is $\phi_{0}(x, y)=y^{4 / 3}$ and $j(x)=1$. For finite width of the current sheet $\phi(x, y) \rightarrow$ $\phi_{1}(y)=y$ as $|x| \rightarrow \infty$, and we expect $\phi(x, y)$ inside the beam, $|x|<a$, to satisfy the inequality $y^{4 / 3} \leq \phi(x, y) \leq$ $y$.

\section{Narrow Beams}

Integrating (1) between $x=0$ and $x=a$, we obtain

$$
-e(y)+\frac{\partial^{2}}{\partial y^{2}} \int_{0}^{a} \phi(x, y) d x=\frac{4}{9} \int_{0}^{a} \frac{j(x)}{\sqrt{\phi}(x, y)} d x .
$$

Eq.(8) can be rewritten as

$$
-e(y)+a \frac{d^{2} \phi\left(x_{1}, y\right)}{d y^{2}}=\frac{4 a j\left(x_{2}\right)}{9 \sqrt{\phi\left(x_{2}, y\right)}},
$$

where $0 \leq x_{1}(y), x_{2}(y) \leq a$. Assuming now that for $a \ll 1$ there is only a small variation in $\phi(x, y)$ for $0 \leq x \leq a$, at least when $y$ is not too close to zero or one, we approximate (9) by setting $\phi\left(x_{1}, y\right)=\phi\left(x_{2}, y\right)=\psi(y)$ and $j\left(x_{2}\right)=j$, where $j$ is a parameter, close to the average current density, which will be determined together with $\phi(x, y)$. We expect that in the limit $a \rightarrow 0, e(y)$, $\psi(y)$ and $a j$ will become independent of $a$ (see section $6)$. We therefore take the equation

$$
-e(y)+a \frac{d^{2} \psi(y)}{d y^{2}}=\frac{4 a j}{9 \sqrt{\psi(y)}}
$$

together with (7) as a suitable approximation for $a \ll 1$ and try to solve them numerically. The boundary conditions for $\psi$ come from (2): $\psi(0)=0, \psi^{\prime}(0)=0, \psi(1)=1$.

An alternate way to arrive at (10), which also gives some information about the beam profile, is to approximate $\phi(x, y)$ for $a \ll 1$ and $y$ not too close to zero or one , as

$$
\phi(x, y) \simeq \psi(y)+\frac{a}{2}\left(1-\frac{x^{2}}{a^{2}}\right) e(y)
$$

so that $e(x, y) \simeq \frac{x}{a} e(y)$. In the same spirit we would have

$$
j(x) \simeq j\left(\eta+\xi \frac{x^{2}}{a^{2}}\right),
$$


where $j(\eta+\xi / 3)$ is the mean current density and $\xi$ represents the relative height of the current wings (expecting $\eta$ to be close to 1 ). Substituting (11), and (12) in (1) we obtain two ordinary equations, namely (10) and

$$
9 \frac{a^{2}-x^{2}}{8 a j} \frac{d^{2} e}{d y^{2}} \approx \frac{\eta+\xi x^{2} / a^{2}}{\sqrt{\psi+e(y)\left(a^{2}-x^{2}\right) / 2 a}}-\frac{1}{\sqrt{\psi}}
$$

The ratio

$$
\zeta(y)=-\frac{e(y) a}{2 \psi(y)}
$$

gives the relative depth of the potential profile (11) at its deepest point $(x=0)$ as a function of $y$.

\section{Numerical Algorithm}

To solve (10) numerically we write $\psi$ in the form

$$
\psi(y)=y^{4 / 3}+\sum_{m=1}^{n} c_{m} f_{m}(y),
$$

where the $f_{m}$ are a set of twice differentiable functions with the properties $f_{m}(0)=f_{m}^{\prime}(0)=f_{m}(1)=0, m=$ $1,2, \ldots, n$ to satisfy the boundary condition $(2)$ for $\psi(y)$.

The boundary electric field is similarly expressed as

$$
e(y)=e_{0}(y)+\sum_{m=1}^{n} c_{m} e_{m}(y) .
$$

For computing $e_{m}(y), m=0,1, . ., n$, we use (6) with the coefficients, given by (5),

$$
a_{k}^{m}=\int_{0}^{1} f_{m}^{\prime}(y) \cos \pi k y d y
$$

After this we apply the direct variational method to minimize the functional $\Phi\left(c_{1}, c_{2}, \ldots, c_{n} ; j\right)$

$$
\Phi=\int_{y_{\min }}^{y_{\max }}\left[e(y)-a \frac{d^{2} \psi(y)}{d y^{2}}+\frac{4 a j}{9 \sqrt{\psi(y)}}\right]^{2} y^{4 / 3} d y
$$

in terms of the parameters $c_{m}$ and $j$. The factor $y^{4 / 3}$ is used in (18) for regularization. "Neutralization" of the nonlinearity of $1 / \sqrt{\psi(y)}$ in (18) is achieved by an iteration procedure where at each step we use parameters $c_{1}, c_{2}, \ldots, c_{n}$ evaluated in the previous step. The functional $\Phi$ thus becomes bilinear in terms of its parameters and we solve repeatedly a linear algebraic system

$$
\frac{\partial \Phi}{\partial j}=0, \quad \frac{\partial \Phi}{\partial c_{m}}=0, m=1,2, . ., n
$$

until its solutions stabilize.

A serious problem in this variational computation is a good choice of the trial functions $f_{m}$. We want in particular the right behavior near the singular points $y=0$ and $y=1$. It is easy to see from $(6)$ that $e(0)=e(1)=0$, but their derivatives are generally divergent. On the other hand if the error in a small neighborhood of these points is not large their overall impact on $e(y), 0<y<1$ is not significant. To eliminate these regions in computing $\Phi$ we set $y_{\min } \approx 0.09$ and $y_{\max } \approx 0.99$ in $(18)$. We monitor the validity of our algorithm by two indicators: (1) the minimum value of $\Phi$ after the iterations get stabilized and (2) by deviations of the left part of Eq. (10) multiplied by $9 \sqrt{\psi(y)} / 4 a$ from the current $j$, at $y=0.1,0.2, \ldots, 0.9$. For flexibility in approximating $\psi(y)$ and in order to keep the matrix of the system (19) away from any degeneracy we choose for $f_{m}(y), m=2,3, \ldots, 10$ a set of partially overlapping functions of finite support. The function $f_{1}(y)$ which corrects the CLL $f_{0}(y)$ for small $y$ is taken of the form $e^{-\beta y} y^{4 / 3}$ with $\beta \sim 40-60$.

To carry out our scheme practically we calculate in advance the partial electric fields $e_{m}(y)$ on a homogeneous grid of 1000 points and then apply the iterations. These usually converge rapidly (after less than 10 steps) while each iteration takes a very short time. Some series converge rather slowly (as $k^{-4 / 3}$ ), but even for a precision of $10^{-9}$, i.e about $10^{5}$ terms in the series, the computation of all partial fields is very fast.

After finding $\psi(y)$ and $e(y)$ the same, but much simpler, procedure can be applied for evaluating the parameters $\eta$ and $\xi$ in (12). We use for Eq.(13) the least squares method, where the weight function in the functional is chosen in such a way as to eliminate the derivatives of $e(y)$, and the computation is done around the middle of the beam.

\section{Results for the current sheet.}

In Table 1 we present the results of the variational procedure described in the last section for different values of $a$. We show there the parameter $j$ which is found by (19) and the mean deviation $\Delta$ of the corresponding quantity, defined in terms of $\psi(y)$ and $e(y)$ in Eq.(10), on the segment $0.1 \leq y \leq 0.9$. The precision of computation is controlled by the ratio $\Phi / \Phi_{0}$ at the stationary point, where

$$
\begin{aligned}
& \Phi_{0}=\left(\frac{4 a j}{9}\right)^{2} \int \frac{y^{4 / 3}}{\psi(y)} d y \\
& \begin{array}{|ccccccc|}
a & 0.2 & 0.1 & 0.05 & 0.02 & 0.01 & 0 \\
j * a & 0.549 & 0.447 & 0.394 & 0.360 & 0.347 & 0.326 \\
\Delta & 0.074 & 0.080 & 0.075 & 0.062 & 0.053 & 0.041 \\
\Phi / \Phi_{0} & .0052 & .0061 & .0055 & .0043 & .0044 & .0067 \\
\xi & 0.042 & 0.032 & 0.021 & 0.009 & 0.004 & \\
\delta & 0.026 & 0.021 & 0.014 & 0.006 & 0.003 &
\end{array} \mid
\end{aligned}
$$

TABLE 1. Flat current sheet

Also shown there are the depth $\xi$ of the current density dip across the beam and the quantity

$$
\delta=1-\eta-\frac{\xi}{3}
$$


which gives the relative difference between the value $j$ computed by solving Eq.(10) and the mean current density $\bar{j}(x)$. The last column in Table 1 exhibits the limiting value of these quantities when $a \rightarrow 0$ using a procedure described below.

\section{The limit $a \rightarrow 0$.}

Our model assumes that the Larmor radii of electrons do not exceed $a$ and this makes the limit $a \rightarrow 0$ physically questionable since this would require the magnetic field to become unreasonably strong. Nevertheless the limit $a \rightarrow 0$ is very interesting both mathematically and practically for evaluation of the prefactor in the scaling law for $j$.

Looking at Eq. (9) we expect that in the limit $a \rightarrow 0$ the term $a j\left(x_{2}\right) \rightarrow \lambda$, independent of $a, \psi\left(x_{2}, y\right) \rightarrow \psi(y)$, and $a \frac{d^{2} \phi(x, y)}{d y^{2}} \rightarrow 0$ except at $y=0, y=1$. The limiting $\psi(y)$ and $e(y)$ will then satisfy the equation

$$
e(y)+\frac{4 \lambda}{9 \sqrt{\psi(y)}}=0
$$

with boundary conditions which we do not know. We therefore solved (20) in a truncated interval $y_{1}=0.09 \leq$ $y \leq 0.998=y_{2}$ using the same routine as before. The result of the computation is presented in the last column of Table 1 . We believe now that by improving and extending the set of trial functions $f_{n}(y)$ one can gradually take $y_{1}, y_{2}$ closer to 0 and 1 respectively, but we think that $\lambda \simeq 0.3$ is a good approximation.

To get an "exact" equation for $\psi$ and $\lambda$ we integrate Eq. (20) over an interval $\left[y_{1}, y_{2}\right] 0<y_{1}<y_{2}<1$ and sum the series in (5) and (6) to yield a nonlinear eigenvalue problem

$$
\frac{9}{4 \pi} \int_{0}^{1} \psi^{\prime}(t) \ln \left|\frac{\cos \pi y_{1}-\cos \pi t}{\cos \pi y_{2}-\cos \pi t}\right| d t=\lambda \int_{y_{1}}^{y_{2}} \frac{d t}{\sqrt{\psi(t)}}
$$

where the boundary conditions are not needed. We postpone investigation of (21) for the future.

\section{Cylindrical beam}

We consider now the more common case of electron beams of a compact cross section when the emitting part of the cathode as well as the cross section of the beam are circular. In cylindrical coordinates Eq. 1 takes the form

$$
\frac{1}{r} \frac{\partial}{\partial r}\left(r \frac{\partial \phi}{\partial r}\right)+\frac{\partial^{2} \phi}{\partial y^{2}}=\frac{4 j(r)}{9 \sqrt{\phi(r, y)}}
$$

with the boundary conditions

$$
\phi(r, 0)=0, \phi(r, 1)=1 ; \frac{\partial \phi}{\partial y}(r, 0)=0, \text { for } r<a
$$

and $j(r)=0$ when $r>a$. Fig. 1 represents now the beam cross section which passes through its axis $r=0$.
Carrying out again a Fourier expansion for $\phi(r, y)$ in the vacuum region $r>a$ yields the potential as a series

$$
\phi(r, y)=y+\frac{2}{\pi} \sum_{k=1}^{\infty} \frac{a_{k}}{k} \frac{K_{0}(\pi k r)}{K_{0}(\pi k a)} \sin \pi k y, r \geq a
$$

where $a_{k}$ can be found by (5) with $\psi(y)=\phi(r, y)$ at $r=a$. Calling now $e(y)$ the radial component of the electric field, at $r=a$, leads to the analogue of (6)

$$
e(y)=-\frac{\partial \phi}{\partial r}(a, y)=2 \sum_{k=1}^{\infty} \frac{K_{1}(\pi k a)}{K_{0}(\pi k a)} a_{k} \sin \pi k y .
$$

Here and in (24) $K_{0}, K_{1}$ are the modified Bessel functions which decay exponentially at infinity.

We can apply the same technique as in (9)-(12) and obtain instead of Eq.(10)

$$
-2 e(y)+a \frac{d^{2} \psi}{d y^{2}}=\frac{4 j a}{9 \sqrt{\psi(y)}} .
$$

Eqs.(13) and (19) for the current and potential profiles are the same. The factor 2 in (26) shows the stronger effect of the surrounding electric fields on the cylindrical beam compared with that on the narrow sheet. The same numerical scheme as in part 4 yields the results shown in Table 2

$\left|\begin{array}{cccccc}a & 0.2 & 0.1 & 0.05 & 0.02 & 0.01 \\ j * a & 5.574 & 5.532 & 5.452 & 5.422 & 5.410 \\ \Delta & 0.090 & 0.069 & 0.052 & 0.037 & 0.036 \\ \Phi / \Phi_{0} & .0051 & .0029 & .0023 & .0026 & .0029 \\ \xi & .1089 & .0566 & .0293 & .0119 & .0059\end{array}\right|$

TABLE 2. Cylindrical beam

The current density behaves like $j \sim 5.4 / a$ and therefore the total beam current becomes proportional to $a$, when $a \ll 1$. The prefactor for cylindrical beams is significantly higher than the one for current sheets because in this case the electron flow is completely surrounded by the vacuum field, but a compact expression like (21) for the limiting parameter $\lambda$ and the boundary potential $\psi$ is impossible here. The profiles of the current density and potential are flatter than in the current sheet.

\section{Discussion}

As already noted the current density $j$ scales approximately as $a^{-1}$ and thus will be large when the beam is narrow with the total current of the sheet becoming independent of the sheet thickness (provided by the unlimited emissivity of the cathode). The current density grows slightly near the beam boundaries, but even for the flat sheet whose thickness is 0.4 , which is not small, this rise is less than $10 \%$ (the evaluation of this quantity is more reliable for $a<0.1$ ). On the other hand when 
$y$ is small the potential across the beam width varies much stronger. In Fig. 2 we show the electric field $e(y)$ for $a=0.1$ and the maximum deviation $\zeta(y)$ (14) of the equipotential surface from the horizontal for $a=0.1$ and $a=0.02$.

When $a \rightarrow 0$ the potential $\psi(y)$ approaches the solution of (21). This function does not differ too much from $y^{4 / 3}$. Only when $y$ is small does $\psi(y)$ increase substantially. It has also an irregular behavior near $y=1$ where $\psi^{\prime \prime}(y) \approx 4 j / 9$, since $e(1)=0$. On the other hand $\psi^{\prime \prime}(y)$ is of order of 1 almost everywhere on the segment $(0,1)$ because if $\psi^{\prime \prime}(y)>C, C \geq 0$ on an interval $y_{1} \leq y \leq y_{2}$ then, using the inequality $\psi^{\prime}(y) \geq 0$, we would get $\psi\left(y_{2}\right)-\psi\left(y_{1}\right)>C\left(y_{2}-y_{1}\right)^{2} / 2$ which, if $C$ is large, will contradict the condition $\psi(y) \leq 1$. Therefore the term $e(y)$ is dominant almost everywhere in the left side of (10) for $a \ll 1$.

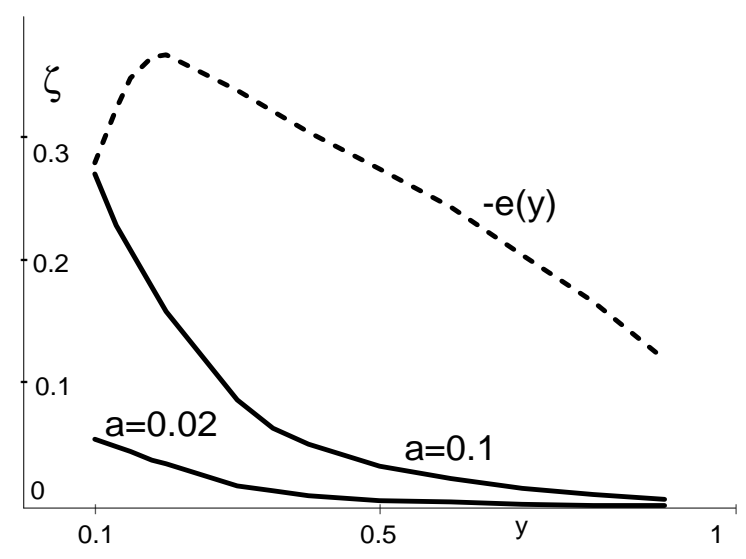

FIG. 2. Current sheet. Plots of $\zeta(y)$ for $a=0.1,0.02$ (solid lines) and $e(y)$ for $a=0.1$ (dashed line)

The equipotential surfaces deviate from horizontal planes only when $y$ is small (in the case of $a=0.01$ this effect is of lower order). Clearly our method of computation, which neglects the $x$-derivatives higher than 2 , is not accurate near $y=0$ where the electron density has a complicated structure in the $x$-direction. We believe nevertheless (see discussion in part 6) that these short intervals do not play a crucial role because the current is limited by the whole space charge distributed between the electrodes.

There is a simple generalization of the parabolic shape assumption used in (11),(12) for $\phi$ and $j(x)$ for larger $a$ [11]. This uses for the approximate solution of Eq.(1) the following ansatz

$$
\phi(x, y)=\psi(y)+e(y) w(x), j(x)=j[\eta-\xi w(x)],
$$

where $w(x)=(\cosh g a-\cosh g x) / k \sinh g a$ and the positive parameter $g$ can be taken from our previous work [9] $(g \approx 3.88)$. Substituting $(27)$ into (1) yields two ordinary differential equations similar to (10) and (13). The advantage of the scheme used here for narrow beams is the transparent relationship between the exact Eq.(9) and our main Eq.(10) which is approximate for $a \neq 0$ but becomes exact, see (20), when $a \rightarrow 0$.

Acknowledgments. Research supported by AFOSR Grant \# F49620-01-0154. and by NSF Grant DMR 01279-26. We thank R. Barker for useful discussions.

[1] A.S.Gilmour, Jr., Microwave Tubes (Artech House, Dedham, MA, 1986); P.T.Kirstein, G.S.Kino, and W.E.Waters, Space Charge Flow (McGraw-Hill, New York, 1967); A.Valfells, D.W.Feldman, M.Virgo, P.G.O'Shea, and Y.Y.Lau, Phys. Plasmas 9, 2377 (2002).

[2] J.W.Luginsland, Y.Y.Lau, R.J.Umstattd, and J.J.Watrous, Phys. Plasmas 9, 2371 (2002); R.J.Umstattd and J.W.Luginsland, Phys. Rev. Lett. 87, 145002 (2001)

[3] J.W.Luginsland, Y.Y.Lau, and R.M.Gilgenbach, Phys. Rev. Lett. 77, 4668 (1996); Y.Y.Lau, Phys. Rev. Lett. 87, 278301 (2001); Y.Y.Lau, P.J.Christenson, and D.Chernin, Physics of Fluids B5, 4486 (1993).

[4] R.J.Umstattd, D.A.Shiffler, C.A.Baca, K.J.Hendricks, T.A.Spencer, and J.W.Luginsland, Proc. SPIE Int. Soc. Opt. Eng. 4031, 185 (2000); D.C.Barnes and R.A.Nebel, Phys. Plasmas 5, 2498 (1998); R.A.Nebel and D.C.Barnes, Fusion Technology 38, 28 (1998).

[5] C.D.Child, Phys. Rev. 32, 492 (1911); I.Langmuir, Phys. Rev. 2, 450 (1913); I.Langmuir and K.B.Blodgett, Phys. Rev. 22, 347 (1923); I.Langmuir and K.B.Blodgett, Phys. Rev. 24, 49 (1924).

[6] J.R.Pierce, Theory and Design of Electron Beams, (Van Nostrand, New York, 1954).

[7] P.W.Hawkes and E.Kasper, Principles of Electron Optics Vol.2 (Academic Press, London, 1989), Chap.46.

[8] J.Rouse et al., in Proceedings of SPIE Vol. 3777, 65 (1999); M.A.Monastyrski, A.G.Murav'ev, and V.A.Tarasov, in Proceedings of SPIE Vol.4187, 2 (1999).

[9] A.Rokhlenko and J.L.Lebowitz, Phys. Rev. Lett. 91, 085002-(1-4) (2003).

[10] M.Abramowitz and A.Stegun (editors), Handbook of Mathematical Functions (Wiley, New York, 1984).

[11] A.Rokhlenko and J.L.Lebowitz, in preparation. 